\title{
Constructing 1D/2D interwoven carbonous matrix to enable high-efficiency sulfur immobilization in Li-S battery
}

\author{
Jiafeng Ruan ${ }^{1,2, \#, ~ H a o ~ S u n ~}{ }^{1, \#}$, Yun Song', Yuepeng Pang ${ }^{1}$, Junhe Yang1, Dalin Sun², Shiyou Zheng ${ }^{1}$ \\ 'School of Materials and Chemistry, University of Shanghai for Science and Technology, Shanghai 200093, China. \\ ${ }^{2}$ Department of Materials Science, Fudan University, Shanghai 200433, China. \\ \#Authors contributed equally.
}

Correspondence to: Prof. Shiyou Zheng, School of Materials and Chemistry, University of Shanghai for Science and Technology, 516 Jungong Road, Shanghai 200093, China. E-mail: syzheng@usst.edu.cn

How to cite this article: Ruan J, Sun H, Song Y, Pang Y, Yang J, Sun D, Zheng S. Constructing 1D/2D interwoven carbonous matrix to enable high-efficiency sulfur immobilization in Li-S battery. Energy Mater 2021;1:100018.

https://dx.doi.org/10.20517/energymater.2021.22

Received: 14 Nov 2021 First Decision: 2 Dec 2021 Revised: 15 Dec 2021 Accepted: 21 Dec 2021 Published: 31 Dec 2021

Academic Editors: Yuping Wu, Jia-Qi Huang Copy Editor: Xi-Jun Chen Production Editor: Xi-Jun Chen

\begin{abstract}
The lithium-sulfur battery is currently considered to be a promising candidate for next-generation energy storage devices. However, its commercial application is severely restricted by rapid capacity decay mainly arising from unavoidable dissolution of intermediate lithium polysulfide of the S-based cathodes. Herein, multifunctional stripped grapheme-carbon nanotubes (SG-CNT) with 1D/2D interwoven and hierarchical pore structure as a promising host to stabilize $\mathrm{S}$ was constructed by cheaper raw materials and a facile strategy. Based on comprehensive analysis, the interwoven network and hierarchical pores along with abundant oxidative functional groups in matrix provided large contact area with S, short transport pathway for electrons/Li-ions, sufficient space to accommodate volumetric change, and superior confinement ability for $\mathrm{S} /$ polysulfides, thus resulting in effectively stabilizing the S cathode with high S loading and increasing its utilization. Therefore, the S@SG-CNT cathodes exhibited a high reversible capacity of $1227 \mathrm{mAh} \mathrm{g}^{-1}$ at $0.1 \mathrm{~A} \mathrm{~g}^{-1}$, excellent cyclability with a capacity of $773 \mathrm{mAh} \mathrm{g}^{-1}$ after 500 cycles at $0.2 \mathrm{~A} \mathrm{~g}^{-1}$, and ultra-long cycling performance with capacity decay less than $0.01 \%$ per cycle at $2 \mathrm{~A} \mathrm{~g}^{-1}$. This facile strategy and unique construction of superior performance cathode provide a new avenue for next commercial application.
\end{abstract}


Keywords: Graphene, carbon, hierarchical pores, cathode, Li-S battery

\section{INTRODUCTION}

The lithium-sulfur (Li-S) batteries have attracted enthusiastic interest as powerful energy storage systems owing to their overwhelmingly high theoretical energy density $\left(2580 \mathrm{Wh} \mathrm{kg}^{-1}\right)$ and gravimetric capacity $\left(1672 \mathrm{mAh} \mathrm{g}^{-1}\right)$. The most incomparable advantages of $\mathrm{S}$ are that it is naturally low cost, abundant, and environmentally friendly, making the Li-S battery one of most hopeful candidates of next-generation battery technology ${ }^{[1-4]}$. Unfortunately, the Li-S battery still presents fast capacity fading, low overall capacity, and high self-discharge, which are attributed to: (1) low S content and large volume change during the lithiation/delithiation process; (2) very poor electrical conductivity induced by insulating of S; and (3) internal "shuttle reaction" arising from the dissolution of lithium polysulfide (LPS) intermediates $\left(\mathrm{Li}_{2} \mathrm{~S}_{\mathrm{x}}, 4 \leq\right.$ $\mathrm{x} \leq 8)$ into organic electrolytes ${ }^{[5-7]}$.

To overcome the low utilization and low loading, encapsulating $S$ into carbonaceous materials with pore structure has been considered as an effective tactic in the past few decades ${ }^{[8]}$. In this case, architecting pore structure is a key for the S and Li-S battery because it directly determines the loading amount and subsequent confinement effect. It is well known that microporous (the pore size $<2 \mathrm{~nm}$ ) can accommodate $S$ and prevent the formation of LPS; however, it is easy to block solid products and eventually limit the penetration of electrolyte during cycling. Mesopores $(2-50 \mathrm{~nm}$ ) have been considered as a hopeful type; however, they cannot simultaneously achieve abundant accommodation of $\mathrm{S}$, effective alleviation of soluble LPS. And fast Li-ion transport in the electrolytes. Macropores (> $50 \mathrm{~nm}$ ) attain the high loading of $\mathrm{S}$ owing to its large pore size, but they are not conducive to retarding the shuttle of LPS $^{[9-11]}$. To overcome these single-sized porous limitations in carbon materials, a hierarchical pore structure, which combines the strengths of the three pore sizes synergistically, could be used. In addition to the size distribution of pores, the existence of abundant pores (especially the large-size pores) in the carbonaceous materials also impacts on the original matrix structure and its conductivity, which can further influence the long-cycling performance $^{[12]}$. It is worth noting that a three dimensional (3D) cross-linked network in the carbonaceous materials, compared with $1 \mathrm{D}$ and $2 \mathrm{D}$ carbon-based materials, can not only ensure structure stability against deformation but also facilitate the electron/ion transport, which can in turn achieve mass transport and fast electrode kinetics ${ }^{[13]}$. Consequently, a hierarchical pore structure and conductive microstructure of carbon materials is desired for Li-S batteries.

More importantly, besides physically confining $\mathrm{S}$ in porous structures, the chemical interaction between the functional carbon matrix and S also exerts a positive impression on the Li-S battery's performance. As described in the relevant literature, the oxidative functional groups (OFG) on the surface of the matrix show strong effects on the polysulfide anions and can trap LPS into the organic electrolyte during the lithiation process ${ }^{[14]}$. For instance, porous structures with a large amount of OFG can improve the capacity retention of $S$ cathodes while limiting the diffusion of LPS into electrolyte, which might be attributed to the strong binding effects between the LPS and the OFG of the active matrix ${ }^{[15]}$. Similarly, Ji et al. ${ }^{[16]}$ reported that the abundant OFG on the surface of the graphene oxide could immobilize $S$, therefore improving the electrochemical performance. Recently, constructing a unique microstructure and introducing polar materials (such as metal oxides/selenides/sulfides and metal atoms) can display a more compact adsorption of LPS than a carbon-based matrix, which can further enhance cycling performance for Li-S batteries ${ }^{[17-21]}$. However, these methods are very complicated. Motivated by the above discussions, using a facile strategy to design a matrix containing hierarchical pores and stable microstructure together with abundant OFG is favorable for Li-S batteries, with the aim that it will exhibit high reversibility and ultra-long cyclic stability of 
$S$ cathodes.

In this study, multifunctional 3D stripped grapheme-carbon nanotubes (denoted as SG-CNT) with hierarchical structure were firstly prepared by a facile oxidation-reduction method, and subsequently an infiltration method was employed to load S to fabricate S@SG-CNT composites at high temperature for Li-S battery cathodes. In the S@SG-CNT composites, the SG-CNT host possesses 1D/2D intrinsic interwoven carbonous matrix [1D CNT skeleton and 2D stripped graphene $(\mathrm{G})$ sheets with covalent C-C bonds], micro-meso-macro hierarchical pores, and abundant oxidative functional groups, providing solid contact with S, short transport pathway for both electrons and Li-ions, sufficient room to accommodate volumetric change, and superior retarding ability for the shuttle of LPS. The S@SG-CNT cathodes containing more than $64 \mathrm{wt} . \% \mathrm{~S}$ present high reversible specific capacity and excellent rate cycling stability, especially a low capacity decay rate of less than $0.01 \%$ per cycle within 2000 cycles at a higher current rate of $2.0 \mathrm{~A} \mathrm{~g} \mathrm{~g}^{-1}$. The systematic results can provide a new insight into the $1 \mathrm{D} / 2 \mathrm{D}$ structural design for matrix host for Li-S batteries.

\section{EXPERIMENTAL}

Synthesis of S@SG-CNT: Firstly, the SG-CNT was synthesized from multi-walled CNT by a modified Hummer's method. Before transferring the oxidized CNT to the hydrothermal reactor, the solution was ultrasonicated for half an hour. After that, this solution was placed into a Teflon-lined stainless-steel autoclave for hydrothermal at $200{ }^{\circ} \mathrm{C}$ for $48 \mathrm{~h}$. Finally, the as-prepared SG-CNT hydrogel was freeze-dried. Then, the S@SG-CNT was prepared by infiltration of the sublimed S powder into SG-CNT in evacuated quartz capsule $\left(\mathrm{m}_{\mathrm{SG}-\mathrm{CNT}}: \mathrm{m}_{\mathrm{S}}=3: 7\right)$ at $700{ }^{\circ} \mathrm{C}$ for $6 \mathrm{~h}$. From that, the S@SG-CNT was obtained after cooling down to room temperature. For comparisons, S@CNT and S@O-CNT were prepared by carbonizing of merging pure $\mathrm{CNT}$ and $\mathrm{O}-\mathrm{CNT}$ with sublimed $\mathrm{S}$, respectively.

Physical characterizations: SEM images were obtained by using a FEI Nova SEM 230 (form Oxford Instruments, named INCA X-Max 80). Deep morphologies and microstructure were characterized by TEM at $200 \mathrm{kV}$ (JEOL Ltd. from Japan). The XRD patterns were recorded on X-ray diffractometer (Rigaku D/MAX-2200/PC) with a Cu Ka radiation at $40 \mathrm{kV}$ and $20 \mathrm{~mA}$. Raman spectra were obtained by a Senterra R200-L. The surface properties were analyzed by XPS (Kratos Axis Ultra DLD). BET and porosity of the assynthesized sample were carried out using a $\mathrm{N}_{2}$ sorption instrument (Micromeritics, ASAP2020). To confirm the S content in S@CNT, S@O-CNT, and S@SG-CNT, thermogravimetric analysis (TGA) was performed with a heating rate of $10^{\circ} \mathrm{C} / \mathrm{min}$ (a Netzsch STA $449 \mathrm{~F} 1$, Germany) and high-purity $\mathrm{N}_{2}$ as the purge gas.

Electrochemical measurements: The electrochemical performance was tested using 2032 half-cell. The composite was mixed with carbon black and polyvinylidene fluoride in a weight ratio of 85:5:10, with Nmethylpyrrolidone solvent as a dispersant. The as-prepared slurry was coated onto an Al foil. After that, the electrodes were punched into circle discs. The battery was assembled in a glovebox (MBRAUN). Before being transferred to the glovebox, the electrodes were further dried in a vacuum at $60{ }^{\circ} \mathrm{C}$ for $24 \mathrm{~h}$. Li metal was used as a counter; Celgard 3501 (Celgard, LLC Corp., USA) was used as a separator; and 1.0 M LiTFSI was dissolved in a mixture of $1,3 \mathrm{DOL} / 1,2 \mathrm{DME}(1: 1 \mathrm{v} / \mathrm{v})$ with $1.0 \% \mathrm{wt} . \mathrm{LiNO}_{3}$ additive was applied as the electrolyte. The electrolyte/S ratios were 11 and $8 \mu \mathrm{L} \mathrm{mg}^{-1}$ for cathodes with $S$ loading of $\sim 2.4 \mathrm{mg} \mathrm{cm}^{-2}$ and higher S loadings $\left(4.1,5.3\right.$, and $\left.6.6 \mathrm{mg} \mathrm{cm}^{-2}\right)$, respectively. Cycling performances were tested in the potential range of 1.5-3.0 V on a LAND CT2001A battery test system (Wuhan Jinnuo Electronics, Ltd.). Cyclic voltammetry (CV) was performed at a scan rate of $0.1 \mathrm{mV} \mathrm{s}^{-1}$ (Gamry Co., Reference 3000, USA). Before electrochemical impedance spectroscopy (EIS) tests, the cell was cycled to the target cycle number at 
2.0 $\mathrm{A} \mathrm{g}^{-1}$. For the $\mathrm{Li}_{2} \mathrm{~S}_{6}$ adsorption test, $0.02 \mathrm{M} \mathrm{Li}_{2} \mathrm{~S}_{6}$ was dissolved in DOL/DME with a volumetric ratio of $1 / 1$.

\section{RESULTS AND DISCUSSION}

Figure 1 displays the schematic diagram of the preparation processes of ultrafine $\mathrm{S}$ particles confined in the CNT, oxidized CNT (O-CNT), and stripped-G CNT (SG-CNT), respectively. First, O-CNT was prepared via a facile oxidation method ${ }^{[22]}$, which can easily introduce abundant OFG and more defects on the outer walls of CNT. After hydrothermal reaction, a black hydrogel can be facilely obtained via a self-assembly condensation reaction. Meanwhile, the C-C bonds around the defects and disorder of the outer CNT walls prefer to break each other, and then the outer walls of CNT are automatically stripped, becoming $2 \mathrm{D} \mathrm{G}$ sheets $^{[23]}$. After that, the $2 \mathrm{D} \mathrm{G}$ sheets can enwrap and string the inner $1 \mathrm{D}$ CNT to become a $1 \mathrm{D} / 2 \mathrm{D}$ intrinsically coupled structure (SG-CNT), and the exposed edges of the broken CNT have a great tendency to produce plentiful oxidative functional groups. Following freeze-drying, we can achieve a 3D SG-CNT sponge with more porous structure, which can facilitate the absorption of $S$ vapor by the strong capillary effect during the following high-temperature infiltration ${ }^{[24]}$. Upon vaporization, S vapor can easily permeate into the empty volume of the SG-CNT matrix by a large driving force at high temperature. To investigate the influence of the unique structure of SG-CNT on the electrochemical performances, the S@SG-CNT, S@CNT, and S@O-CNT composites were prepared by combination of merging the CNT and O-CNT with sublimed S under the same condition, respectively. In contrast to the S@CNT and S@O-CNT composites, it was hypothesized that S@SG-CNT would present excellent electrochemical performance owing to the crosslinked skeletons and pore structure promoting interactions between ultrafine $S$ and the SG-CNT matrix.

The morphologies of the three matrices were elucidated by SEM and TEM. At the suspended solution stage, the O-CNT with oxygen-functional groups can uniformly disperse as compared with the pristine CNT [Figure 2A and B]. Moreover, CNTs in the obtained CNT powder and O-CNT powder are both separated, as shown in the HRTEM characterization in Figure 2D and E. After hydrothermal treatment, a lightweight and porous carbon sponge with cross-linked structure was easily obtained by the self-assembly process [Figure $2 \mathrm{C}$ ]. To further investigate the effect of oxidation and hydrothermal process on the self-assembly process of the carbon matrix, three samples were characterized by HRTEM. It can be seen that the pristine CNT has a smooth wall with a thickness of $\sim 4.11 \mathrm{~nm}$ [Figure 2D]. After oxidation, the surface of O-CNT becomes rough, which indicates abundant defects and active sites are rooted into the surface walls of each CNT [Figure 2E]. During hydrothermal process, the surface morphology of SG-CNT, as shown in Figure 2F and Supplementary Figure 1, exhibited a 1D/2D interwoven structure interconnected with $1 \mathrm{D}$ CNT and 2D stripped-G, implying a stable covalent linkage between $C N T$ and $G$ via condensation reaction ${ }^{[3]}$. This $1 \mathrm{D} / 2 \mathrm{D}$ unique structure can also be demonstrated by Raman patterns, as shown in Supplementary Figure 2. This unique $3 \mathrm{D}$ porous $\mathrm{CNT}$-bridged stripped-G building blocks can effectively facilitate fast ions/electrons diffusion and promote the electrical conductivity. Not that abundant porous structures and edge defects are presented on the $3 \mathrm{D}$ crumpled SG-CNT matrix, which can favor for the infiltration of sulfur while tolerating the volume expansion during the cycling process.

XPS measurements were used to investigate the chemical compositions as well as the oxidation state of the elements. As depicted in Supplementary Figure 3, the CNT, O-CNT, and SG-CNT samples are all mainly composed of $\mathrm{O}$ and $\mathrm{C}$ elements, while the atomic ratio of $\mathrm{O} / \mathrm{C}$ in the O-CNT and SG-CNT samples decrease from 0.288 to 0.154 [Supplementary Table 1], implying that the OFG were assembled under high temperature $\left(200^{\circ} \mathrm{C}\right)$ and pressure during the hydrothermal process ${ }^{[25]}$. The O 1s XPS spectra of the O-CNT and SG-CNT in Supplementary Figure 4 exhibit four peaks at 534.3, 533.2, 532.0, and 531.2 eV, which are assigned to the carboxylic, epoxide, hydroxyl, and carbonyl groups, respectively ${ }^{[26]}$. Interestingly, the main O-containing functional groups in the SG-CNT change to epoxide groups (occupying 7.73 at.\%; see 


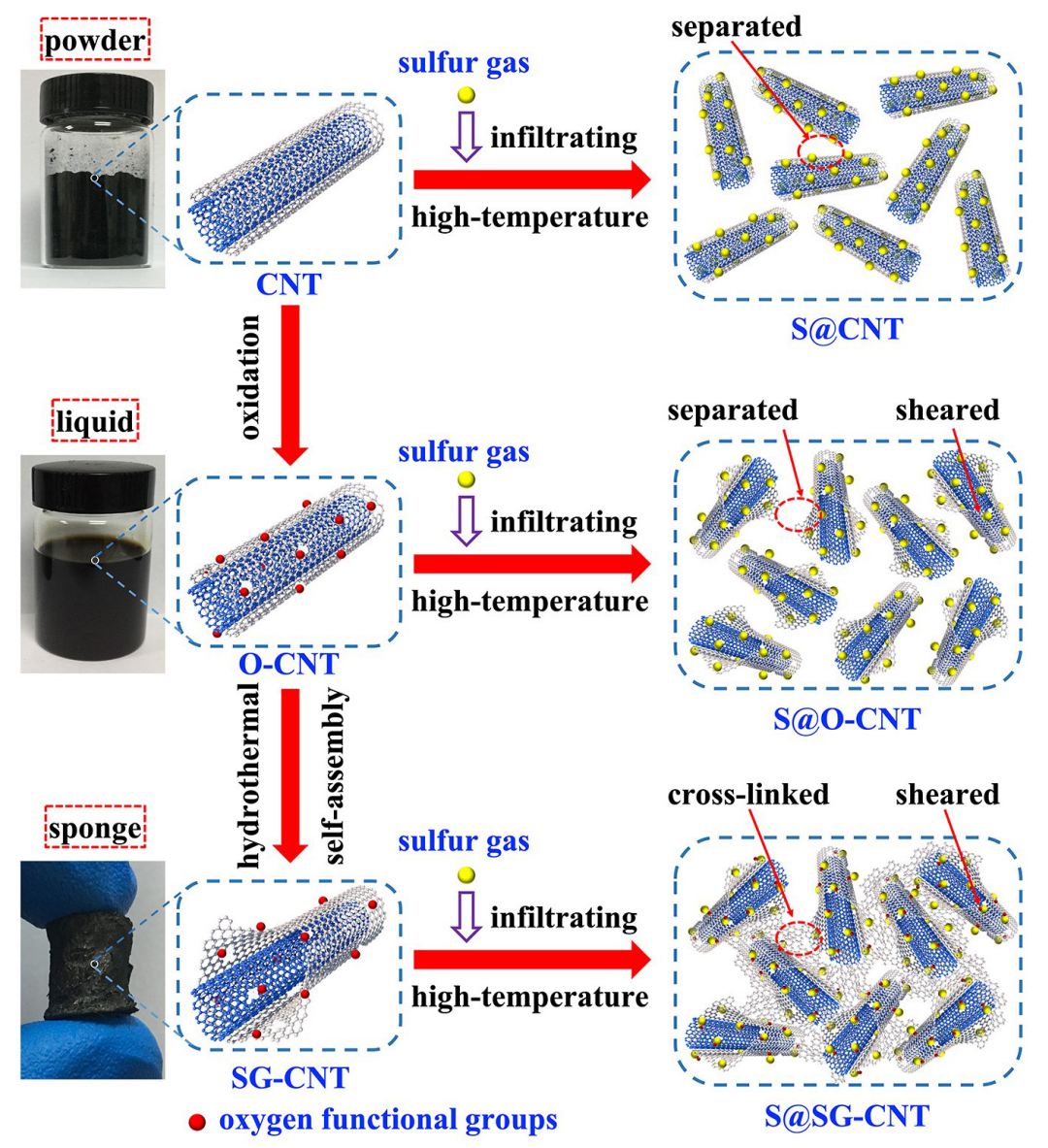

Figure 1. Schematic diagrams of the preparation processes of S@CNT, S@O-CNT, and S@SG-CNT, respectively.

Supplementary Table 2) accompanied by decrease of the carboxylic (1.12 at.\%) and hydroxyl (3.69 at.\%) groups after hydrothermal reaction. Such dynamic change of the O-containing functional groups in the carbon framework of SG-CNT shows a stable covalent linkage between carbon material. For instance, the OFG can improve the wettability in the inert surface area and expose more active sites to absorb $S^{[27]}$. Besides, all O-containing functional groups readily trap S species ${ }^{[16]}$. Moreover, the hydroxyl groups, which were abundant after high-temperature vapor infiltration, show stronger chemical interaction to the polysulfide anions than do the epoxide groups ${ }^{[26,28]}$. This is due to the fact that abundant hydroxyl groups $(-\mathrm{OH})$ on the surface of SG-CNT can create an asymmetrical charge distribution on the S cluster, leading to greater polarization, thus strengthening the electrostatic interaction between S and the SG-CNT matrix ${ }^{[26,29]}$. From the above TEM and XPS analyses, the structure and schematic configuration of $\mathrm{C}$ atoms and $\mathrm{OFG}$ in the three samples are shown in Figure $2 \mathrm{G}$. Benefiting from the unique $1 \mathrm{D} / 2 \mathrm{D}$ tube-bridged sheets architectures and more hydroxyl functional groups contained in SG-CNT, the migration of LPS into organic electrolyte during the redox reactions is effectively suppressed. Besides, the stable chemical bonds could improve the electronic conductivity and further enhance the electrochemical performance of the composites $^{[30]}$.

The S@CNT, S@O-CNT, and S@SG-CNT composites were obtained with high-temperature vapor infiltration method. The $\mathrm{S}$ contents were measured by TGA [Figure 3A], which indicated that the $\mathrm{S}$ contents in S@CNT, S@O-CNT, and S@SG-CNT are 63.75\%,64.53\%, and 64.36\%, respectively. The equal S mass 

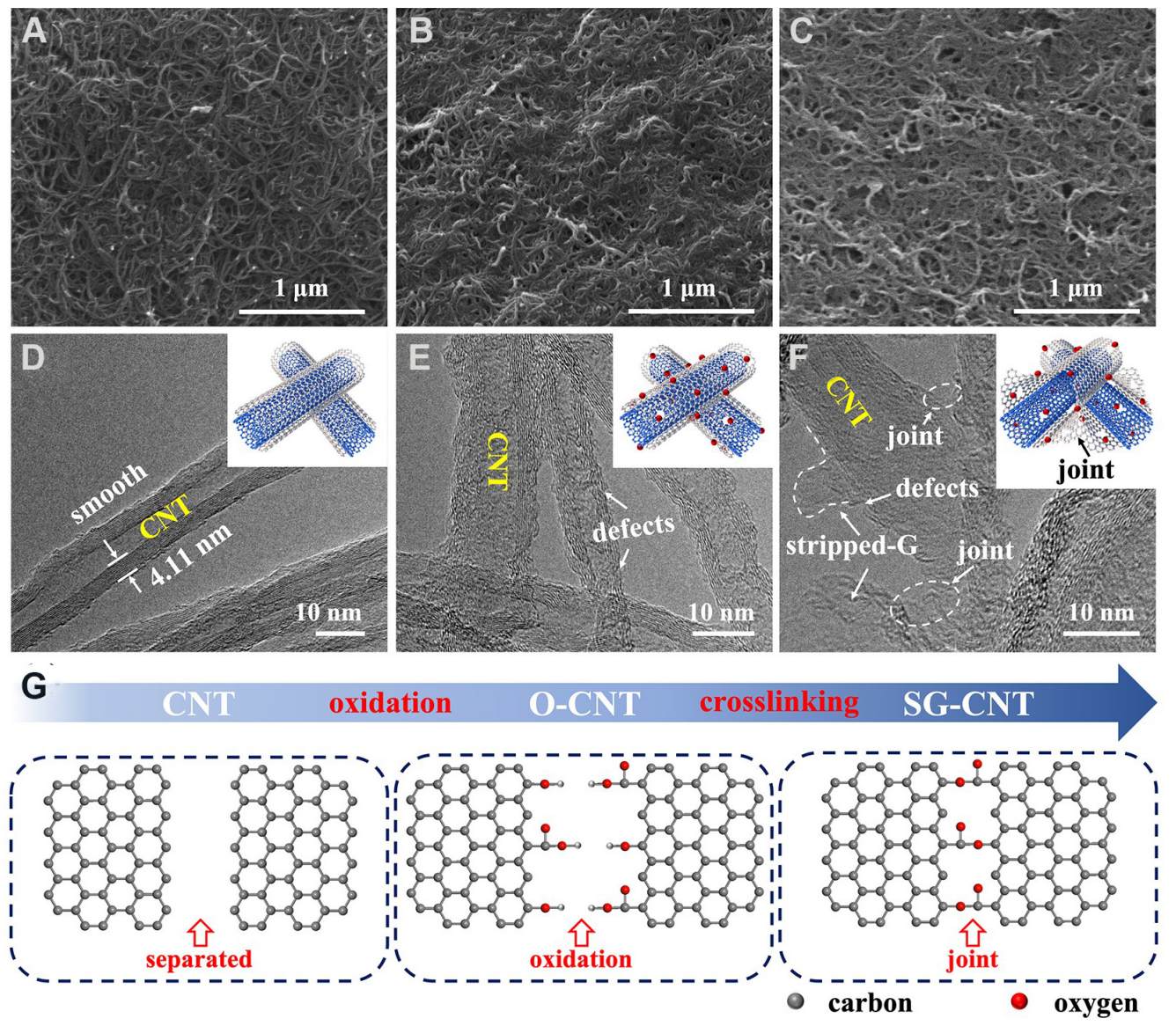

Figure 2. (A-C) SEM images of (A) CNT, (B) O-CNT, and (C) SG-CNT; (D-F) TEM images of (D) CNT, (E) O-CNT, and (F) SG-CNT; and (G) schematics of oxygen functional groups in CNT (left), O-CNT (middle), and SG-CNT (right).

content in these three composites is convenient to compare the battery performance without concerning the variation of the S loading.

The phase composition of the S@CNT, S@O-CNT, and S@SG-CNT samples were measured. The XRD peaks of crystalline S are observed in Figure 3B for S@CNT and S@O-CNT, while the peak intensity of S is drastically weakened for S@SG-CNT sample, which suggests that the ultrafine S nanoparticles are evenly distributed in the nanoscale pores and exclusively confined between the carbon layers in the SG-CNT host $^{[31]}$. The existence of a strong intimate interplay between S and SG-CNT host led to a relatively poor crystallinity compared to the S@CNT and S@O-CNT. This was further evidenced by Raman analysis [Supplementary Figure 5], in which three composites all show two pronounced peaks around $1350 \mathrm{~cm}^{-1}(\mathrm{D}$ band, due to heteroatom defects) and $1590 \mathrm{~cm}^{-1}$ ( $\mathrm{G}$ band, due to "sp ${ }^{3}$ graphitic" framework), and their intensity ratio $\left(\mathrm{I}_{\mathrm{D}} / \mathrm{I}_{\mathrm{G}}\right)$ is a measure of the amount of disorder of the carbon matrix ${ }^{[16]}$. Compared with the S@CNT and S@O-CNT, the $\mathrm{I}_{\mathrm{D}} / \mathrm{I}_{\mathrm{G}}$ ratio of S@SG-CNT is much higher (1.13), indicating that plenty of defects and vacancies are present in S@SG-CNT, which could not only promote the diffusion of Li ions but also provide more active sites for improving the electrochemical performance effectively ${ }^{[32,33]}$.

The pore size distribution and specific surface area (SSA) of three S-implanted composites and corresponding matrix were evaluated by $\mathrm{N}_{2}$ adsorption tests. As shown in Figure $3 \mathrm{C}$, a hierarchical structure with micro-meso-macro pore size distribution was observed in the S@SG-CNT matrix. Besides, the SSA and 

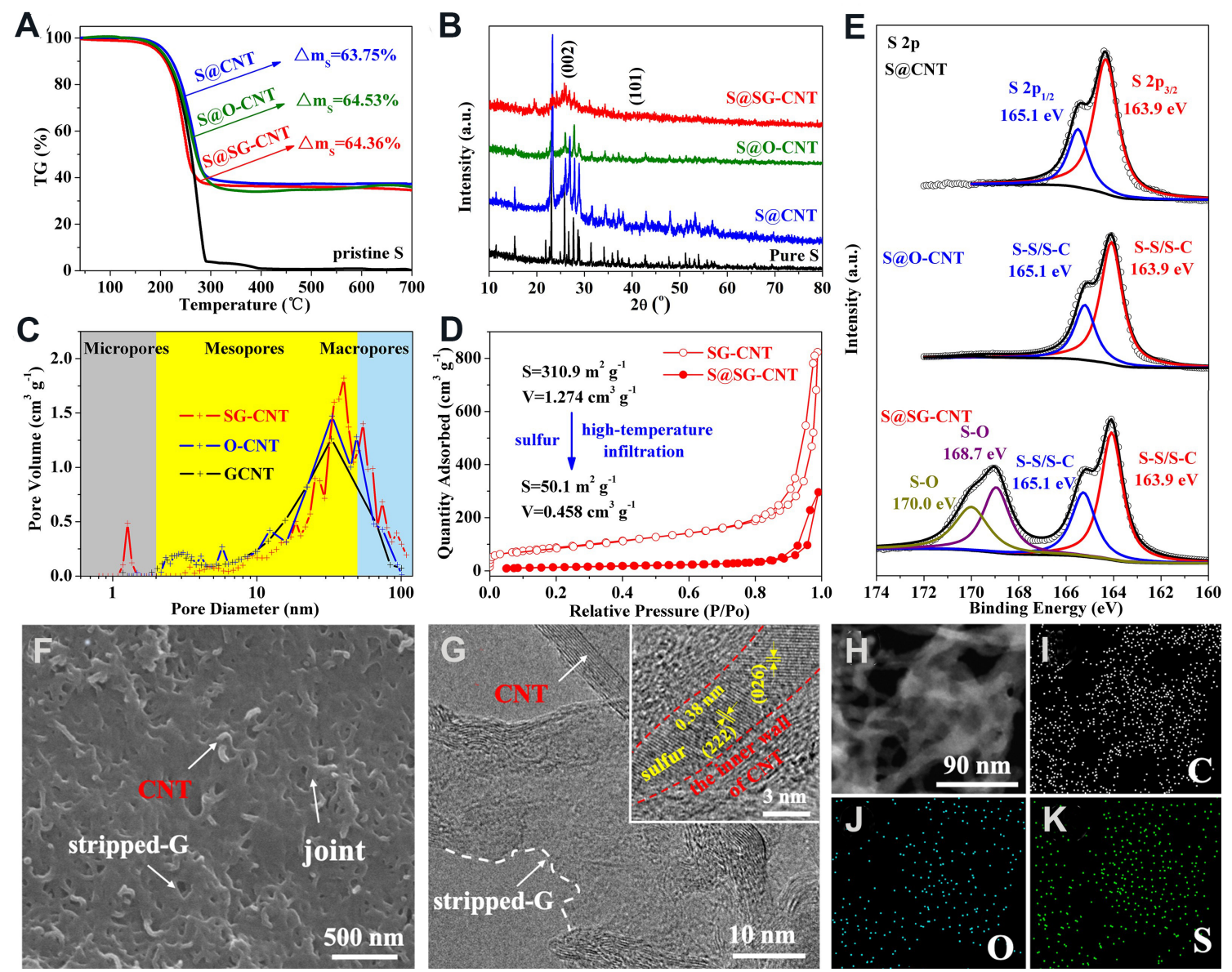

Figure 3. (A) TGA patterns of S@CNT, S@O-CNT, and S@SG-CNT; (B) XRD and (C) pore size distribution of CNT, O-CNT, and SGCNT; (D) $N_{2}$ adsorption isotherms of SG-CNT and S@SG-CNT; (E) high-resolution XPS spectra of S 2p of three composites; (F) SEM images of S@SG-CNT; (G) TEM images of S@SG-CNT; and (H-K) STEM image of the representative S@SG-CNT (H) and corresponding $\mathrm{C}(\mathrm{I}), \mathrm{O}(\mathrm{J})$, and $\mathrm{S}(\mathrm{K})$ element mapping.

the pore volume of the S@CNT, S@O-CNT, and S@SG-CNT composites in Figure 3D and Supplementary Figure 6 are much smaller than their corresponding matrix. For instance, the pore volume and SSA of SGCNT is $1.274 \mathrm{~cm}^{3} \mathrm{~g}^{-1}$ and $310.9 \mathrm{~m}^{2} \mathrm{~g}^{-1}$, while the corresponding values decreased to $0.458 \mathrm{~cm}^{3} \mathrm{~g}^{-1}$ and $50.1 \mathrm{~m}^{2} \mathrm{~g}^{-1}$ after loading $\mathrm{S}$ (see Figure 3D). We therefore believe that $\mathrm{S}$ was loaded into most of the inner spaces of the SG-CNT host, especially the smaller S molecules $(1<\mathrm{n} \leq 4)$ can be loaded in the micropores ${ }^{[31]}$. In addition, the mesopores $(2-50 \mathrm{~nm})$ can accommodate the volume expansion of $S$ and are valuable for trapping the dissolved LPS, strictly prohibiting capacity fade, while the macropores can facilitate the charge transportation owing to their large pore size and increase the active site of the material ${ }^{[34]}$.

To further elucidate the interaction between matrix and S, the chemical environments of three composites were also elucidated by XPS analysis. As shown in Supplementary Figure 7 and Supplementary Table 3, the O elements could only be detected for S@SG-CNT and could hardly be found in S@O-CNT, which indicates that most OFG were removed from the O-CNT after hydrothermal process, while the robust C-O bonds could be maintained in SG-CNT. We therefore believe that the hydrothermal process helps oxygencontaining functional groups anchor on carbon matrix with strong chemical bonds. As shown in Supplementary Figure 8, the deconvolution plot of the C 1s XPS spectra for S@SG-CNT shows four peaks at 
289.0, 286.7, 285.6, and $284.7 \mathrm{eV}$, which correspond to $\mathrm{O}-\mathrm{C}=\mathrm{O}, \mathrm{C}=\mathrm{O}, \mathrm{C}-\mathrm{O} / \mathrm{C}-\mathrm{S}$, and $\mathrm{C}=\mathrm{C} / \mathrm{C}-\mathrm{C}$ bonds, respectively. The occurrence of C-S bonds suggests that $\mathrm{S}$ atoms were doped in SG-CNT and formed a robust bond during the high-temperature treatment ${ }^{[35]}$. The O 1s XPS spectrum for the S@SG-CNT [Supplementary Figure 9] is deconvoluted into three peaks at 531.2, 533.2, and $534.3 \mathrm{eV}$, which are assigned to $\mathrm{C}=\mathrm{O}, \mathrm{C}-\mathrm{O}-\mathrm{C}$ (epoxide), and $\mathrm{HO}-\mathrm{C}=\mathrm{O}$ groups (carboxyl), respectively ${ }^{[26]}$. However, in contrast with the SG-CNT matrix, the peak of $\mathrm{O} 1 \mathrm{~s}$ centered at $532.0 \mathrm{eV}$ was shifted, accompanying a new S-O band that appears upon annealing [Supplementary Table 4$]^{[36]}$. In addition, these new peaks of S-O are also clearly observed in the S 2p XPS spectra (168.7 and 170.0 eV, in Figure 3E) of S@SG-CNT, which directly provide evidence of the existence of chemical bonds between $\mathrm{S}$ and oxygen functional groups ${ }^{[37]}$. The XPS comparison of these two shows that the formation of the $\mathrm{C}-\mathrm{O}-\mathrm{C}$ bond facilitates the formation of strong $\mathrm{S}-\mathrm{O}$ bonds in the process of compounding sulfur (see Supplementary Figure 10). These above analyses display that the ultrafine $\mathrm{S}$ particles maintain intimate solid contact with the carbon matrix (SG-CNT) through C-S/O-S bonding, which is valuable for immobilizing $\mathrm{S}$ and eliminating the shuttle effect during the discharge/charge process in Li-S batteries.

The morphology evolution of S@CNT, S@O-CNT, and S@SG-CNT composites were also investigated. As shown in Figure $3 \mathrm{~F}$ and Supplementary Figure 11, there was no obvious structure change for the three composites after the $S$ impregnation. In comparison, the 1D/2D interwoven structure was observed in S@SG-CNT, while it vanished in S@CNT and S@O-CNT. Notably, bulk S particles [Supplementary Figure 12] could not be clearly detected in the SEM image of S@SG-CNT, which may be attributed to the fact that the S molecules were uniformly and ultra-finely confined for the S@SG-CNT composite. This above phenomenon could also be proved by the TEM image of S@SG-CNT. In contrast with the TEM images of S@CNT and S@O-CNT in Supplementary Figure 13, the 3D CNT-bridged G structure can be recognized in S@SG-CNT, which can reach excellent properties by keeping the matrix structure stability during the cycling process and improving the electrical conductivity. Figure $3 \mathrm{G}$ shows the $\mathrm{S}$ could encapsulate in carbon nanotubes at high temperature and pressure. The adjacent fringe spacing is around 0.34 and 0.38 $\mathrm{nm}$, corresponding to the (026) and (038) plane of S, respectively, implying that a robust intimate contact existed between S and the SG-CNT matrix, which complies with the XPS results. The EDS mapping of S@SG-CNT was used to confirm the distribution of elements. Figure $3 \mathrm{H}-\mathrm{K}$ shows that the three elements (C, O, and S) in S@SG-CNT were uniformly distributed in the large region.

To demonstrate the role of unique structural benefits of the SG-CNT sponge with 3D cross-linked hierarchical pore structure and the interaction of S-O play in enhancing cathode performance of the Li-S battery, the electrochemical properties of the serial materials were measured and compared. Figure $4 \mathrm{~A}$ depicts the initial five CV of the S@SG-CNT electrode under a voltage range of 1.5-3.0 V (vs. Li/ $\left.\mathrm{Li}^{+}\right)$. Three reduction peaks $(\sim 2.35, \sim 2.0$, and $\sim 1.7 \mathrm{~V})$ were observed in cathodic scan, which were ascribed to the reduction of high-order polysulfides $\left(\mathrm{Li}_{2} \mathrm{~S}_{\mathrm{x}}, 4 \leq \mathrm{x} \leq 8\right)$ to low-order polysulfides (such as $\mathrm{Li}_{2} \mathrm{~S}_{4}, \mathrm{Li}_{2} \mathrm{~S}_{2}$, and $\left.\mathrm{Li}_{2} \mathrm{~S}\right)^{[38]}$. In the subsequent reverse scan, one broad oxidation bump (at 2.3-2.7 V) was observed, which corresponded to the reduction of $\mathrm{S}$ to high-order polysulfides ${ }^{[39]}$. Besides, both the peak positions and areas in CV were reproducible in the sequence cycling, showing high reversibility of multi-step conversion. Similar electrochemical behaviors were also observed in the S@CNT and S@O-CNT electrodes [Supplementary Figure 14], except for a broad reversible reduction peak (at $\sim 1.7 \mathrm{~V}$ ) relevant to the reduction of the smaller $\mathrm{S}$ molecules $(1<\mathrm{n} \leq 4)$ in the micropores to insoluble $\mathrm{Li}_{2} \mathrm{~S}_{2} / \mathrm{Li}_{2} \mathrm{~S}$ in the S@SG$\mathrm{CNT}^{[31]}$, which vanished in the S@CNT and S@O-CNT composites after the initial cycle, implying that the stability of 3D cross-linked, micropores structure and the S-O chemical bonds in S@SG-CNT were effective for immobilizing $\mathrm{S}$ and alleviating the soluble LPS during the succeeding cycling process, thus preventing the loss of $S$. 

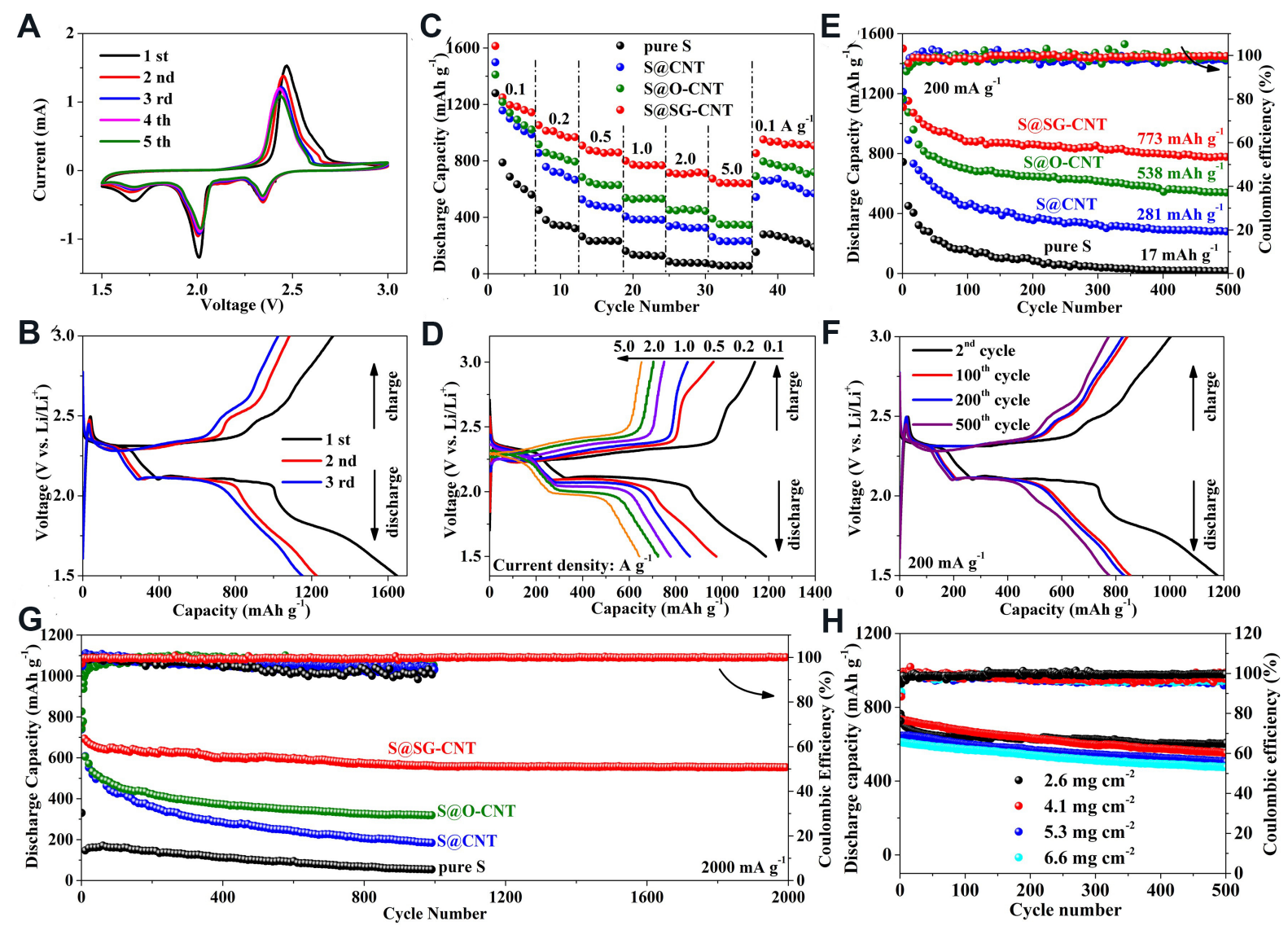

Figure 4. Electrochemical performance of the electrode based on the sulfur mass: (A) Cyclic voltammetry curves of S@SG-CNT electrodes at a scan rate of $0.1 \mathrm{mV} \mathrm{s}^{-1}$; (B) initial three discharge/charge profiles of S@SG-CNT cathode at a current density of $0.1 \mathrm{~A} \mathrm{~g}^{-1}$; (C) rate-cycle performance; (D) the second charge/discharge curves of the S@SG-CNT at 0.1, 0.2, 0.5, 1.0, 2.0, and 5.0 A g ${ }^{-1}$, respectively; (E) cycling performance at $200 \mathrm{~mA} \mathrm{~g}^{-1}$; (F) the 2nd, 100th, 200th, and 500th charge/discharge curves of the S@SG-CNT at $0.2 \mathrm{~A} \mathrm{~g}^{-1}$, respectively; $(\mathrm{G})$ long-term cycling performance; and $(\mathrm{H})$ long cycling life of the $\mathrm{S} @ S G-C N T$ with four different mass loading of $\mathrm{S}$ at $2000 \mathrm{~mA} \mathrm{~g}^{-1}$.

Figure 4B exhibits the initial three discharge/charge profiles of S@SG-CNT cathodes under $0.1 \mathrm{~A} \mathrm{~g}^{-1}$. The three discharge plateaus $(\sim 2.3, \sim 2.0$, and $\sim 1.7 \mathrm{~V})$ and one charge plateau $(\sim 2.35 \mathrm{~V})$ were displayed for the S@SG-CNT composites in the first cycle, which is accordance with the above observation of CV. The S@SG-CNT delivers an ultra-high discharge/charge capacity of 1647.5/1321.1 $\mathrm{mAh} \mathrm{g}^{-1}$, which can give a high Coulombic efficiency (CE) of $80.2 \%$ in the first cycle. Upon cycling, the plateau at $\sim 1.7 \mathrm{~V}$ disappeared, consistent with the CV results. Meanwhile, a high discharge capacity was sustained at $1226.6 \mathrm{mAh} \mathrm{g}^{-1}$ in the second cycle, and the CE exceeded to 95\%. In comparison, the pristine S, S@CNT, and S@O-CNT cathodes only displayed discharge capacities $\left(1130,1220\right.$, and $1346 \mathrm{mAh} \mathrm{g}^{-1}$, respectively) in the first cycle and corresponding CE (65.4\%, 79.3\%, and 71.5\%, respectively) [Supplementary Figure 15]. The low capacities and CE were due to the large capacity loss of the pristine S, S@CNT, and S@O-CNT cathodes, caused by the dissolved polysulfides due to the unsuitable architectures of the S@CNT and S@O-CNT composites. Thus, it is safe to say that the excellent battery performance and the high initial CE of the S@SG-CNT composites are the result of the unique $1 \mathrm{D} / 2 \mathrm{D}$ interwoven structure and the strong chemical interaction of S-Ocontaining functional groups involving the SG-CNT matrix. 
In addition, the S@SG-CNT cathode displayed excellent capability under various current densities. As shown in Figure 4C, high reversible specific capacities of 1201, 991, 860, and $772 \mathrm{mAh} \mathrm{g}^{-1}$ for the S@SGCNT cathodes could be obtained at $0.1,0.2,0.5$, and $1.0 \mathrm{~A} \mathrm{~g} \mathrm{~g}^{-1}$, respectively. More importantly, excellent discharge capacities of 720 and $655 \mathrm{mAh} \mathrm{g}^{-1}$ could still be achieved for the S@SG-CNT electrodes even up to ultra-high C-rates of 2.0 and $5.0 \mathrm{~A} \mathrm{~g}^{-1}$, respectively. Following that, when the current density returned to $0.1 \mathrm{~A} \mathrm{~g}^{-1}$ after 40 cycles, an excellent discharge capacity of $911 \mathrm{mAh} \mathrm{g}^{-1}$ could be still maintained, which suggest that S@SG-CNT shows a solid structure for endurance with various current densities, even at 5.0 $\mathrm{A} \mathrm{g}^{-1}$. The rate-cycling capacities of pure S, S@CNT, and S@O-CNT cathodes were lower than that of S@SG-CNT. This is reason that the rapid electronic and ionic transfer can achieve fast electrochemical kinetics to further obtain excellent capacity performance at higher C-rates ${ }^{[40]}$. The outstanding rate performance is even superior to that of some previous studies (see Supplementary Figure 16) ${ }^{[31,41,42]}$. Figure 4D shows the charge/discharge profiles of the S@SG-CNT composites at various rates from 0.1 to $2.0 \mathrm{~A} \mathrm{~g}^{-1}$. In addition to the typical voltage plateaus of $\mathrm{S}$ cathode at 2.35 and $2.0 \mathrm{~V}$, the discharge plateau at $\sim 1.7 \mathrm{~V}$ disappeared in the initial cycle when the loading current was up to $1.0 \mathrm{~V}$, which could be related to the limited redox reaction kinetics at the high current densities.

To further evaluate the structural of the S@SG-CNT composite structure, the long cycling performance of the three composites were investigated. As shown in Figure 4E, the S@SG-CNT composites exhibited a very high reversible capacity of $1309.5 \mathrm{mAh} \mathrm{g}^{-1}$ at $0.2 \mathrm{~A} \mathrm{~g}^{-1}$ and then reached $880 \mathrm{mAh} \mathrm{g}^{-1}$ at around the 100th cycle after undergoing the initial activation procedure. Even up to 500 cycles, the reversible capacity of the S@SG-CNT still maintained $773 \mathrm{mAh} \mathrm{g}^{-1}$, yielding a capacity retention of around 87.8\%. Upon cycling, the corresponding CE tended to increase to $100 \%$ after 50 cycles, reflecting the excellent reversible capacity and low influence of the electrochemical shuttle effect. In contrast with the S@SG-CNT composites, the S@CNT and S@O-CNT cathodes showed very low discharge capacities of 281 and $538 \mathrm{mAh} \mathrm{g}^{-1}$ under the same current density $\left(200 \mathrm{~mA} \mathrm{~g}^{-1}\right)$ after 500 cycles, much lower than that of the S@SG-CNT composites. In addition, the S@SG-CNT material also displayed excellent cycling performance at low E/S ratio (see Supplementary Figure 17). The S@SG-CNT composites exhibited better performance than some previous works (see Table 1) ) $^{[17-21,43-49]}$. In addition, the typical reproducible charge/discharge curves in Figure $4 \mathrm{~F}$ imply the highly reversible stability of the S@SG-CNT composites. In addition, S@SG-CNT also showed excellent cyclability at a high current density. As shown in Figure 4G, the S@SG-CNT composites displayed an initial capacity of $743.1 \mathrm{mAh} \mathrm{g}^{-1}$ and maintained $551.2 \mathrm{mAh} \mathrm{g}^{-1}$ with CE close to $100 \%$ even after 2000 cycles, while the S@CNT and S@O-CNT cathodes only showed 184 and $318 \mathrm{mAh} \mathrm{g}^{-1}$ under the same current density (2000 mA g ${ }^{-1}$ ) after 1000 cycles, implying that S@SG-CNT exhibits excellent rate performance. The improved rate capabilities of S@SG-CNT is attribute to the collaborative effects including the good electrical conductivity, which emanate from the unique "one in two" structure with CNT-bridged stripped G interwoven and the effective inhibition of "shuttle effect" due to the hierarchical pores and the strong S-O interaction via OFG in the smart 3D SG-CNT building blocks. To reveal the practical application of the S@SG-CNT composite, the electrochemical properties of the electrodes with high mass loading of S were investigated. Figure 4H shows the long cycling life of the S@SG-CNT composite with four different mass loadings of S $\left(2.6,4.1,5.3\right.$, and $\left.6.6 \mathrm{mg} \mathrm{cm}^{-2}\right)$ at $2.0 \mathrm{~A} \mathrm{~g}^{-1}$. As shown in Figure 4H, the S@SG-CNT composite with a mass loading of $2.6 \mathrm{mg} \mathrm{cm}^{-1}$ displayed an initial capacity of $743.1 \mathrm{mAh} \mathrm{g}^{-1}$ and maintained $603 \mathrm{mAh} \mathrm{g}^{-1}$ with CE of $99.9 \%$ after 500 cycles, while the other three high mass loading $(4.1,5.3$, and $6.6 \mathrm{mg} \mathrm{cm}^{-2}$ ) electrodes also showed high capacities of 554, 510, and $480 \mathrm{mAh} \mathrm{g}^{-1}$ after 500 cycles. Moreover, the polarization of the S@SG-CNT material did not change significantly with the increase of the loading (see Supplementary Figure 18). Additionally, the lithium metal anode also exhibited good cycling performance under high current density and capacity condition (see Supplementary Figure 19). These results imply that S@SG-CNT with different mass loading of S exhibits excellent performance for future practical applications. 
Table 1. Comparison of the electrochemical property of the S@SG-CNT cathode and other S-based materials previously reported in the related literature in Li-S batteries

\begin{tabular}{llllll}
\hline Materials & S loading $\left(\mathbf{m g ~ c m}^{-2}\right)$ & Current density & Cycle number & After cycles specific capacity $\left(\mathbf{m A h ~}^{-1}\right)$ & Ref. $^{-1}$ \\
\hline S@SG-CNT & 2.6 & $0.2 \mathrm{~A} \mathrm{~g}^{-1}$ & 500 & 773 & This work \\
Sulfur cathode & 1.2 & $0.5 \mathrm{C}$ & 250 & 905 & {$[43]$} \\
SPAN & 1.5 & $0.1 \mathrm{Ag}^{-1}$ & 100 & $\sim 500$ & {$[44]$} \\
S/PCMSs & 2.0 & $0.5 \mathrm{C}$ & 700 & 489 & {$[45]$} \\
CoSe $/$ /G & 1.0 & $4 \mathrm{C}$ & 500 & 503 & {$[17]$} \\
PC@G/S & - & $0.5 \mathrm{C}$ & 1000 & 721.9 & {$[46]$} \\
S/CP@NCNT@CoS & 3 & $1.3 \mathrm{Ag}^{-1}$ & 400 & 680 & {$[18]$} \\
CNT@TiO $2-\mathrm{S}-\mathrm{S}$ & 2.2 & $0.2 \mathrm{C}$ & 100 & 769 & {$[19]$} \\
S/G & 1.5 & $1 \mathrm{C}$ & 250 & 772 & {$[47]$} \\
V-S-80 & 2 & $0.5 \mathrm{C}$ & 200 & 770 & {$[48]$} \\
ZIF-67-S-PPy-60\% & - & $0.1 \mathrm{C}$ & 200 & $\sim 450$ & {$[20]$} \\
HNPC-900-65S & - & $0.5 \mathrm{C}$ & 500 & 788 & {$[49]$} \\
S/TiN-VN@CNFs & 1.7 & $0.2 \mathrm{C}$ & 100 & 950 & {$[21]$} \\
\hline
\end{tabular}

To investigate the mechanism of the stable cycling performance of the S@SG-CNT composites, EIS measurements were carried out. Figure 5A exhibits the Nyquist plots before and after various cycling at 2.0 $\mathrm{A} \mathrm{g}^{-1}$ for the S@SG-CNT composite. All Nyquist diagrams with a quasi-semicircle at the middle frequency region are connected with the charge transfer resistance $\left(\mathrm{R}_{\mathrm{ct}}\right)$, while a sloping line at the low frequency region in the Nyquist plots is related to the Warburg diffusion resistance $\left(\mathrm{R}_{\mathrm{w}}\right)^{[50,51]}$. According to the fitted results, we can easily obtain the equivalent circuit (Figure 5A, inset), the $\mathrm{R}_{\mathrm{ct}}$ of S@SG-CNT sharply decreased from 132 to $34.1 \Omega$ after three cycles [Supplementary Table 5], implying the improvement of charge transportation though the electrode interface due to the initial activation. This process is beneficial for improving the delivery capacity of the 3D electrode and cycling performance. Besides, the $\mathrm{R}_{\mathrm{ct}} \mathrm{of} \mathrm{S} @ \mathrm{SG}$ CNT composites are quite stable up to 1000 cycles, revealing a smart intimate contact and stable interface between the electrolytes and $3 \mathrm{D}$ electrode architectures, contributing to the superior electrochemical performance of the S@SG-CNT composite.

Sweep voltammetry analysis was carried out to further understand the superior electrochemical kinetics of the S@SG-CNT in Li-S cells. Supplementary Figure 20A and B and Figure 5B display the CV curves of the S@CNT, S@O-CNT, and S@SG-CNT electrodes at various scan rates $\left(0.2-1.0 \mathrm{mV} \mathrm{s} \mathrm{s}^{-1}\right)$, respectively. With the increasing of the scan rate, the S@SG-CNT cathode shows the least variation of Peaks 1-3 followed by S@CNT and S@O-CNT, demonstrating that the polarization of S@SG-CNT is smaller than those of S@CNT and S@O-CNT. The relationship between the peak current (i) and scan rate $(v) i s i=a v^{b[52]}$. In Figure 5C and D, the b-values of the S@SG-CNT are 0.61, 0.52, and 0.67 at Peaks 1-3, respectively, which are higher than those of S@CNT and S@O-CNT [Supplementary Figure 20C and D]. In addition, the diffusion $\mathrm{Li}^{+}$ion rate $\left(\mathrm{D}_{\mathrm{Li}+}\right)$ can be qualitatively measured according the Randles-Sevcik equation $\left(\mathrm{I}_{\mathrm{p}}=2.69 \times\right.$ $\left.10^{5} \mathrm{n}^{3 / 2} \mathrm{~A} \mathrm{D}_{\mathrm{Li}+}^{1 / 2} \mathrm{v}^{1 / 2} \mathrm{C}_{\mathrm{Li}+}\right)^{[53]}$ based on the above sweep voltammetry analysis, where $\mathrm{I}_{\mathrm{p}}$ is the strongest peak current, $\mathrm{A}$ is the area of electrolyte $\left(1.13 \mathrm{~cm}^{2}\right), \mathrm{v}$ is the scan rate, $\mathrm{C}_{\mathrm{Li}+}$ is the concentration of $\mathrm{Li}^{+}, \mathrm{n}$ is the transferring electron numbers, and $\mathrm{D}_{\mathrm{Li}+}$ is the diffusion coefficient. The value of $\mathrm{D}_{\mathrm{Li}+}$ is determined from the slope of the linear relations between $\mathrm{I}_{\mathrm{p}}$ and $\mathrm{v}^{1 / 2}$ [Figure 5E and Supplementary Figure 20E]. After calculation, the slope values of S@SG-CNT were 0.821, 1.447, and 2.017 and the corresponding $\mathrm{D}_{\mathrm{Li}+}$ were $2.861 \times 10^{-7}$, $7.978 \times 10^{-7}$, and $1.388 \times 10^{-6} \mathrm{~cm}^{2} \mathrm{~s}^{-1}$ for the three peaks, respectively, which are all larger than those of S@CNT and S@O-CNT, suggesting the fastest intercalation/de-intercalation of $\mathrm{Li}^{+}$in S@SG-CNT (see Figure $5 \mathrm{~F})$. 

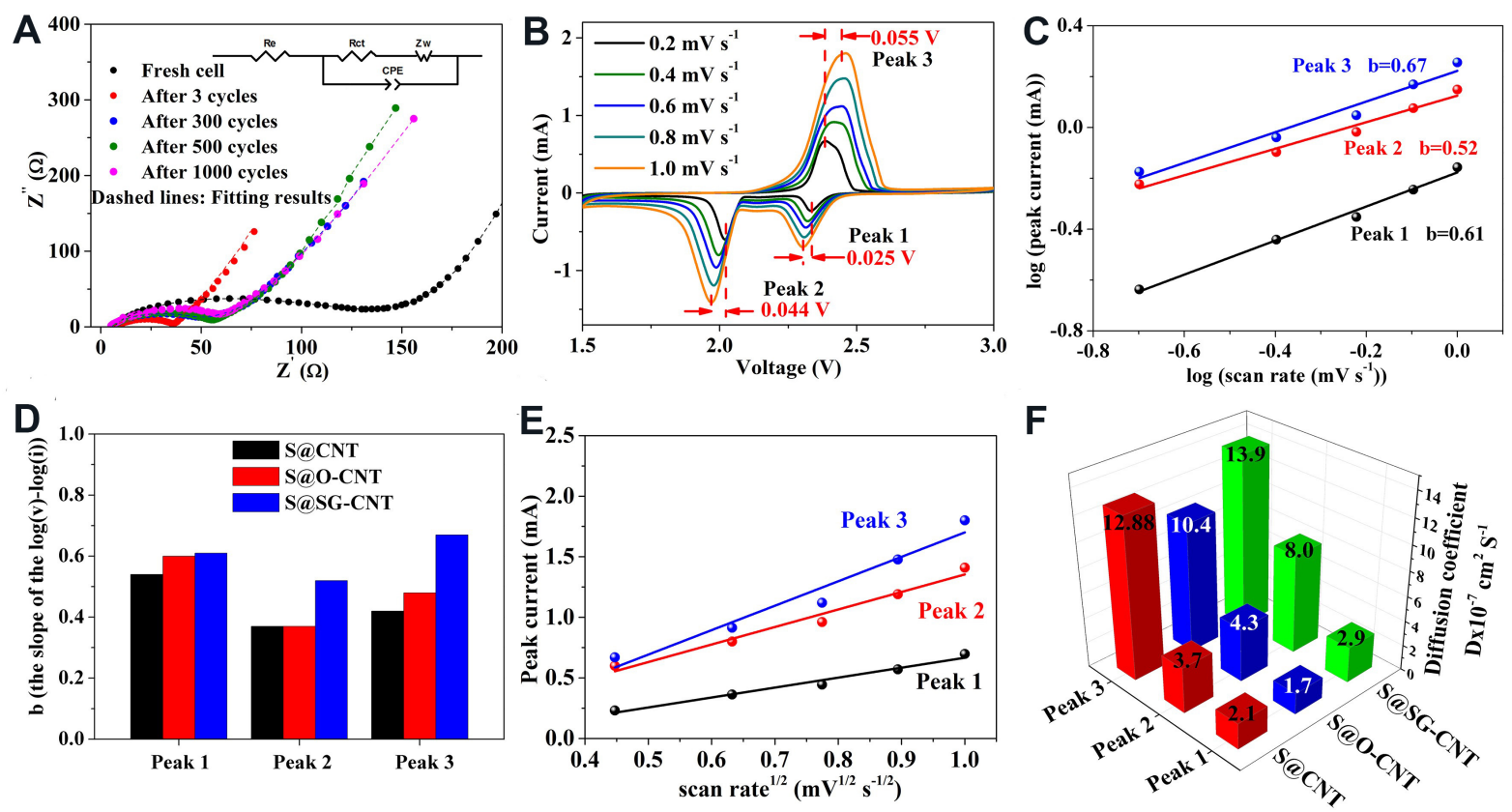

Figure 5. (A) Nyquist plot of the S@SG-CNT before and after cycling with the inset showing the equivalent circuit used for data fitting; (B) the cyclic voltammetry of S@SG-CNT at various scan rates; (C) b-values plotted of the S@SG-CNT for Peaks 1-3; (D) b-values plotted of the three composites; (E) linear fits of the peak current of the S@SG-CNT for three peaks; and (F) Li ${ }^{+}$ion diffusion coefficient for three samples.

Furthermore, the four-point probe method was employed to investigate the influence of the unique $1 \mathrm{D}$ CNT/2D-stripped-G structure on the electrical conductivity of the CNT, O-CNT, and SG-CNT matrices. As shown in Figure 6A, the electronic conductivity of SG-CNT $\left(7.9 \mathrm{~S} \mathrm{~cm}^{-1}\right)$ was the highest, followed by GCNT $\left(3.3 \mathrm{~S} \mathrm{~cm}^{-1}\right)$ and $\mathrm{O}-\mathrm{CNT}\left(2.5 \mathrm{~S} \mathrm{~cm}^{-1}\right)$. This result strongly supports that the $3 \mathrm{D}$ cross-linked structure in the S@SG-CNT can enable fast electron/ion transfer through the 3D robust building blocks.

To analyze the structural properties of the S@SG-CNT after undergoing 2000 cycles, TEM was also employed. As shown in Figure 6B, the S@SG-CNT composite still maintained its initial 1D tube@2D sheets interconnected morphological structure at the current density of $2.0 \mathrm{~A} \mathrm{~g}^{-1}$ after 2000 cycles, indicating its morphology was not damaged during the electrochemical cycles. In addition, the $\mathrm{S}$ content [Figure $6 \mathrm{C}$ ] was not obviously changed after long time cycling, further suggesting that the strong chemical interaction between S and SG-CNT matrix could immobilize $S$ and alleviate the shuttle effect during the discharge/charge process in Li-S batteries and further contribute to a high stable cycling battery performance. To further explore the influence of the different matrices on the trapping capability of LPS, the $\mathrm{Li}_{2} \mathrm{~S}_{6}$ adsorption test was performed. As shown in Figure $6 \mathrm{D}$, the solution with SG-CNT was entirely decolored after $0.5 \mathrm{~h}$, while the solutions with CNT and O-CNT showed less discoloration, demonstrating that SG-CNT with abundant OFG, especially the hydroxyl functional groups, exhibited an excellent binding capability of LPS compared to CNT and O-CNT. Based on the above analysis, the S@SG-CNT frameworks with 3D cross-linked, hierarchical pore structure not only serve as electron channels for fast electrochemical response but also enhance the interaction between $\mathrm{S}$ and matrix, thereby leading to superior electrochemical kinetics compared to those of S@CNT and S@O-CNT, as illustrated in Figure 6E and Supplementary Figure 21. Besides the novel structure for battery performance, the chemical interaction between S and matrix in the S@SG-CNT composites can alleviate the diffusion of LPS into the organic electrolyte and induce the chemical adsorption of LPS, which contributes to a high stable cycling battery 

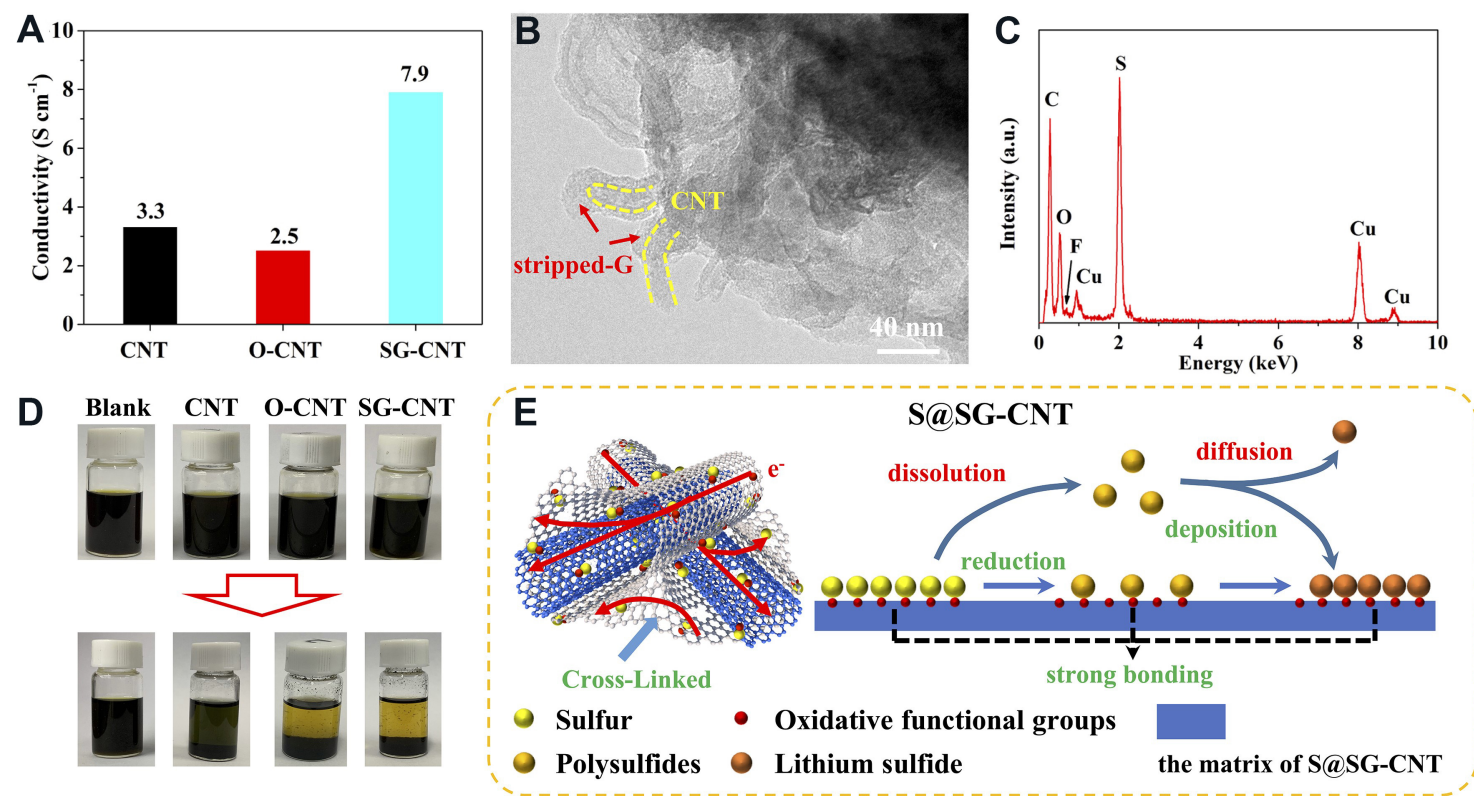

Figure 6. (A) Electrical conductivity of three matrices; (B) TEM image and (C) EDS spectrum of S@SG-CNT after 2000 cycles; (D) visualized adsorption of $\mathrm{Li}_{2} \mathrm{~S}_{6}$ solution; and $(\mathrm{E})$ schematic illustration of the electrochemical reaction mechanism of S@SG-CNT.

performance, superior to those of S@CNT and S@O-CNT composites due to their poor affinity for S/polysulfides/lithium sulfide ${ }^{[5-59]}$. To further improve the electrochemical performance, this unique carbon matrix can be composited with a highly catalytically active material as a S host for Li-S batteries ${ }^{[21,48]}$.

\section{CONCLUSION}

In summary, a 3D SG-CNT matrix with hierarchical pore and abundant oxidative functional group structure was successfully prepared via an oxidation-assembly-reduction method. The unique 3D SG-CNT matrix was used as a smart building block to load $\mathrm{S}$ through a high-temperature infiltration technique to form S@SG-CNT cathode composites for Li-S battery. The S@SG-CNT cathodes with high S loading presented high reversible specific capacity and excellent rate cycling stability. The exceptional electrochemical performances of S@SG-CNT electrodes in Li-S battery is because: (1) the 1D/2D interwoven structure (1D CNT and $2 \mathrm{D}$ stripped $-\mathrm{G}$ ) can provide excellent electrical conductivity, ensuring the rapid electrochemical response and shorten $\mathrm{Li}^{+}$diffusion distance; (2) the hierarchical pores are not only suitable for high S loading but also effectively control the shuttle effect and buffer the volume change of S and structural expansion/shrinkage during long cycling process; and (3) the existence of oxidative functional groups, especially the hydroxyl groups, and the robust S-O chemical bonds on the carbon matrix surface can effectivity alleviate the diffusion of LPS into the organic electrolyte during the redox reactions. It is believed that the facile introduction of the hierarchical pore to carbonaceous host materials can pave a promising avenue for a high-performance Li-S battery.

\section{DECLARATIONS}

\section{Authors' contributions}

Conceptualization, formal analysis, investigation, writing - original draft, review, and editing: Ruan J

Investigation, writing - original draft: Sun $\mathrm{H}$

Formal analysis, writing - review and editing: Song Y 
Formal analysis, investigation, writing - review and editing: Pang Y

Resources, writing - review and editing: Yang J

Supervision, resources, writing - review and editing: Sun D

Supervision, investigation, resources, writing - review and editing: Zheng $S$

\section{Availability of data and materials}

Not applicable.

\section{Financial support and sponsorship}

We gratefully acknowledge the support of the National Natural Science Foundation of China (51971146, 51971147 and 52171218), the Shanghai Municipal Science and Technology Commission (21010503100), the Innovation Program of Shanghai Municipal Education Commission (2019-01-07-00-07-E00015), the General Program of Natural Science Foundation of Shanghai (20ZR1438400), and the Shanghai Rising-Star Program (20QA1407100), and the Postdoctoral Innovation Talents Support Program of China (BX202 1067). We also acknowledge the support of Shanghai Outstanding Academic Leaders Plan and the Guangxi Key Laboratory of Information Materials (Guilin University of Electronic Technology, 201017-K).

\section{Conflicts of interest}

All authors declared that there are no conflicts of interest.

\section{Ethical approval and consent to participate}

Not applicable.

\section{Consent for publication}

Not applicable.

\section{Copyright}

(c) The Author(s) 2021.

\section{REFERENCES}

1. Tan G, Xu R, Xing Z, et al. Burning lithium in $\mathrm{CS}_{2}$ for high-performing compact $\mathrm{Li}_{2} \mathrm{~S}$-graphene nanocapsules for Li-S batteries. Nat Energy 2017;2:17090. DOI

2. Pang Q, Liang X, Kwok CY, Nazar LF. Advances in lithium-sulfur batteries based on multifunctional cathodes and electrolytes. Nat Energy 2016;1:16132. DOI

3. Ding Y, Kopold P, Hahn K, van Aken PA, Maier J, Yu Y. Facile solid-state growth of 3D well-interconnected nitrogen-rich carbon nanotube-graphene hybrid architectures for lithium-sulfur batteries. Adv Funct Mater 2016;26:1112-9. DOI

4. Lin L, Pei F, Peng J, et al. Fiber network composed of interconnected yolk-shell carbon nanospheres for high-performance lithiumsulfur batteries. Nano Energy 2018;54:50-8. DOI

5. Li Z, Zhang J, Guan B, Wang D, Liu LM, Lou XW. A sulfur host based on titanium monoxide@carbon hollow spheres for advanced lithium-sulfur batteries. Nat Commun 2016;7:13065. DOI PubMed PMC

6. Dong Q, Shen R, Li C, et al. Construction of soft base tongs on separator to grasp polysulfides from shuttling in lithium-sulfur batteries. Small 2018;14:e1804277. DOI PubMed

7. Lu K, Zhang H, Gao S, Ma H, Chen J, Cheng Y. Manipulating polysulfide conversion with strongly coupled $\mathrm{Fe}_{3} \mathrm{O}_{4}$ and nitrogen doped carbon for stable and high capacity lithium-sulfur batteries. Adv Funct Mater 2019;29:1807309. DOI

8. Lim WG, Jo C, Cho A, et al. Approaching ultrastable high-rate Li-S batteries through hierarchically porous titanium nitride synthesized by multiscale phase separation. Adv Mater 2019;31:e1806547. DOI PubMed

9. Li Z, Yuan L, Yi Z, et al. Insight into the electrode mechanism in lithium-sulfur batteries with ordered microporous carbon confined sulfur as the cathode. Adv Energy Mater 2014;4:1301473. DOI

10. Ding B, Yuan C, Shen L, Xu G, Nie P, Zhang X. Encapsulating sulfur into hierarchically ordered porous carbon as a high-performance cathode for lithium-sulfur batteries. Chemistry 2013;19:1013-9. DOI PubMed

11. Schuster J, He G, Mandlmeier B, et al. Spherical ordered mesoporous carbon nanoparticles with high porosity for lithium-sulfur batteries. Angew Chem Int Ed Engl 2012;51:3591-5. DOI PubMed

12. Pei F, An T, Zang J, et al. From hollow carbon spheres to N-doped hollow porous carbon bowls: rational design of hollow carbon host for Li-S batteries. Adv Energy Mater 2016;6:1502539. DOI 
13. Share K, Cohn AP, Carter R, Rogers B, Pint CL. Role of nitrogen-doped graphene for improved high-capacity potassium ion battery anodes. ACS Nano 2016;10:9738-44. DOI PubMed

14. Zhou G, Yin LC, Wang DW, et al. Fibrous hybrid of graphene and sulfur nanocrystals for high-performance lithium-sulfur batteries. ACS Nano 2013;7:5367-75. DOI PubMed

15. Demir-Cakan R, Morcrette M, Nouar F, et al. Cathode composites for Li-S batteries via the use of oxygenated porous architectures. $J$ Am Chem Soc 2011;133:16154-60. DOI PubMed

16. Ji L, Rao M, Zheng H, et al. Graphene oxide as a sulfur immobilizer in high performance lithium/sulfur cells. J Am Chem Soc 2011;133:18522-5. DOI PubMed

17. Yuan H, Peng H, Li B, et al. Conductive and catalytic triple-phase interfaces enabling uniform nucleation in high-rate lithium-sulfur batteries. Adv Energy Mater 2019;9:1802768. DOI

18. Yang X, Gao X, Sun Q, et al. Promoting the transformation of $\mathrm{Li}_{2} \mathrm{~S}_{2}$ to $\mathrm{Li}_{2} \mathrm{~S}$ : significantly increasing utilization of active materials for high-sulfur-loading Li-S batteries. Adv Mater 2019;31:e1901220. DOI

19. Wang Y, Zhang R, Chen J, et al. Enhancing catalytic activity of titanium oxide in lithium-sulfur batteries by band engineering. $A d v$ Energy Mater 2019;9:1900953. DOI

20. Geng P, Cao S, Guo X, et al. Polypyrrole coated hollow metal-organic framework composites for lithium-sulfur batteries. J Mater Chem A 2019;7:19465-70. DOI

21. Yao Y, Wang H, Yang H, et al. A dual-functional conductive framework embedded with TiN-VN heterostructures for highly efficient polysulfide and lithium regulation toward stable Li-S full batteries. Adv Mater 2020;32:e1905658. DOI PubMed

22. Hummers WS, Offeman RE. Preparation of graphitic oxide. J Am Chem Soc 1958;80:1339. DOI

23. Chen L, Jin X, Wen Y, et al. Intrinsically coupled 3D nGs@CNTs frameworks as anode materials for lithium-ion batteries. Chem Mater 2015;27:7289-95. DOI

24. Zhu Y, Wen Y, Fan X, et al. Red phosphorus-single-walled carbon nanotube composite as a superior anode for sodium ion batteries. ACS Nano 2015;9:3254-64. DOI PubMed

25. Byon HR, Gallant BM, Lee SW, Shao-horn Y. Role of oxygen functional groups in carbon nanotube/graphene freestanding electrodes for high performance lithium batteries. Adv Funct Mater 2013;23:1037-45. DOI

26. Wu Z, Wang D, Ren W, et al. Anchoring hydrous $\mathrm{RuO}_{2}$ on graphene sheets for high-performance electrochemical capacitors. $A d v$ Funct Mater 2010;20:3595-602. DOI

27. Yang J, Ju Z, Jiang Y, et al. Enhanced capacity and rate capability of nitrogen/oxygen dual-doped hard carbon in capacitive potassiumion storage. Adv Mater 2018;30:1700104. DOI PubMed

28. Wang Z, Dong Y, Li H, et al. Enhancing lithium-sulphur battery performance by strongly binding the discharge products on aminofunctionalized reduced graphene oxide. Nat Commun 2014;5:5002. DOI PubMed

29. Bagri A, Mattevi C, Acik M, Chabal YJ, Chhowalla M, Shenoy VB. Structural evolution during the reduction of chemically derived graphene oxide. Nat Chem 2010;2:581-7. DOI PubMed

30. Ruan J, Yuan T, Pang Y, et al. Nitrogen and sulfur dual-doped carbon films as flexible free-standing anodes for Li-ion and Na-ion batteries. Carbon 2018;126:9-16. DOI

31. Mi K, Jiang Y, Feng J, Qian Y, Xiong S. Hierarchical carbon nanotubes with a thick microporous wall and inner channel as efficient scaffolds for lithium-sulfur batteries. Adv Funct Mater 2016;26:1571-9. DOI

32. Xu Y, Ruan J, Pang Y, et al. Homologous strategy to construct high-performance coupling electrodes for advanced potassium-ion hybrid capacitors. Nanomicro Lett 2020;13:14. DOI PubMed PMC

33. Lin Y, Gao M, Zhu D, Liu Y, Pan H. Effects of carbon coating and iron phosphides on the electrochemical properties of LiFePO4/C. $J$ Power Sources 2008;184:444-8. DOI

34. Yao X, Ke Y, Ren W, et al. Defect-rich soft carbon porous nanosheets for fast and high-capacity sodium-ion storage. Adv Energy Mater 2019;9:1803260. DOI

35. Zheng $\mathrm{S}$, Chen $\mathrm{Y}, \mathrm{Xu} \mathrm{Y}$, et al. In situ formed lithium sulfide/microporous carbon cathodes for lithium-ion batteries. ACS Nano 2013;7:10995-1003. DOI PubMed

36. Cui X, Xie Z, Wang Y. Novel $\mathrm{CoS}_{2}$ embedded carbon nanocages by direct sulfurizing metal-organic frameworks for dye-sensitized solar cells. Nanoscale 2016;8:11984-92. DOI PubMed

37. Zhang L, Ji L, Glans PA, Zhang Y, Zhu J, Guo J. Electronic structure and chemical bonding of a graphene oxide-sulfur nanocomposite for use in superior performance lithium-sulfur cells. Phys Chem Chem Phys 2012;14:13670-5. DOI PubMed

38. Xu R, Lu J, Amine K. Progress in mechanistic understanding and characterization techniques of Li-S batteries. Adv Energy Mater 2015;5:1500408. DOI

39. Lin Z, Liang C. Lithium-sulfur batteries: from liquid to solid cells. J Mater Chem A 2015;3:936-58. DOI

40. Xu Y, Zhang C, Zhou M, et al. Highly nitrogen doped carbon nanofibers with superior rate capability and cyclability for potassium ion batteries. Nat Commun 2018;9:1720. DOI PubMed PMC

41. Bian Z, Yuan T, Xu Y, et al. Boosting Li-S battery by rational design of freestanding cathode with enriched anchoring and catalytic Nsites carbonaceous host. Carbon 2019;150:216-23. DOI

42. Liu S, Li J, Yan X, et al. Superhierarchical cobalt-embedded nitrogen-doped porous carbon nanosheets as two-in-one hosts for highperformance lithium-sulfur batteries. Adv Mater 2018;30:e1706895. DOI PubMed

43. Li Y, Wang W, Liu X, et al. Engineering stable electrode-separator interfaces with ultrathin conductive polymer layer for high-energydensity Li-S batteries. Energy Storage Materials 2019;23:261-8. DOI

44. Xing X, Li Y, Wang X, Petrova V, Liu H, Liu P. Cathode electrolyte interface enabling stable Li-S batteries. Energy Storage Materials 
2019;21:474-80. DOI

45. Liu S, Zhao T, Tan X, et al. 3D pomegranate-like structures of porous carbon microspheres self-assembled by hollow thin-walled highly-graphitized nanoballs as sulfur immobilizers for Li-S batteries. Nano Energy 2019;63:103894. DOI

46. Xu H, Liu Y, Bai Q, Wu R. Discarded cigarette filter-derived hierarchically porous carbon@graphene composites for lithium-sulfur batteries. J Mater Chem A 2019;7:3558-62. DOI

47. Li Q, Song Y, Xu R, et al. Biotemplating growth of nepenthes-like N-doped graphene as a bifunctional polysulfide scavenger for Li-S batteries. ACS Nano 2018;12:10240-50. DOI PubMed

48. Wu F, Lv H, Chen S, et al. Natural vermiculite enables high-performance in lithium-sulfur batteries via electrical double layer effects. Adv Funct Mater 2019. DOI

49. Li Z, Xiao Z, Wang S, Cheng Z, Li P, Wang R. Engineered interfusion of hollow nitrogen-doped carbon nanospheres for improving electrochemical behavior and energy density of lithium-sulfur batteries. Adv Funct Mater 2019;29:1902322. DOI

50. Xing Z, Li G, Sy S, Chen Z. Recessed deposition of TiN into N-doped carbon as a cathode host for superior Li-S batteries performance. Nano Energy 2018;54:1-9. DOI

51. Wu Y, Gao M, Li X, Liu Y, Pan H. Preparation of mesohollow and microporous carbon nanofiber and its application in cathode material for lithium-sulfur batteries. J Alloys Compd 2014;608:220-8. DOI

52. Cao B, Zhang Q, Liu H, et al. Graphitic carbon nanocage as a stable and high Power anode for potassium-ion batteries. Adv Energy Mater 2018;8:1801149. DOI

53. Guo D, Ming F, Su H, et al. MXene based self-assembled cathode and antifouling separator for high-rate and dendrite-inhibited Li-S battery. Nano Energy 2019;61:478-85. DOI

54. Peng H, Huang J, Zhao M, et al. Nanoarchitectured graphene/CNT@Porous carbon with extraordinary electrical conductivity and interconnected micro/mesopores for lithium-sulfur batteries. Adv Funct Mater 2014;24:2772-81. DOI

55. Niu S, Lv W, Zhang C, et al. One-pot self-assembly of graphene/carbon nanotube/sulfur hybrid with three dimensionally interconnected structure for lithium-sulfur batteries. J Power Sources 2015;295:182-9. DOI

56. Feng L, Zhu Z, He Y, et al. Superfast and solvent-free core-shell assembly of sulfur/carbon active particles by hail-inspired nanostorm technology for high-energy-density Li-S batteries. Journal of Energy Chemistry 2022;65:565-73. DOI

57. Peng H, Hou T, Zhang Q, et al. Strongly coupled interfaces between a heterogeneous carbon host and a sulfur-containing guest for highly stable lithium-sulfur batteries: mechanistic insight into capacity degradation. Adv Mater Interfaces 2014;1:1400227. DOI

58. Xiao Z, Yang Z, Nie H, Lu Y, Yang K, Huang S. Porous carbon nanotubes etched by water steam for high-rate large-capacity lithiumsulfur batteries. J Mater Chem A 2014;2:8683-9. DOI

59. Sun H, Li Z, Xia S, Pang Y, Yang J, Zheng S. High-performance lithium-sulfur battery enabled by jointing cobalt decorated interlayer and polyethyleneimine functionalized separator. $J$ Alloys Compd 2021;888:161459. DOI 